\title{
Modelling Cellular Processes using Membrane Systems with Peripheral and Integral Proteins
}

\author{
Matteo Cavaliere \\ The Microsoft Research - University of Trento Centre for Computational and Systems \\ Biology \\ Sean Sedwards \\ The Microsoft Research - University of Trento Centre for Computational and Systems \\ Biology
}

This is a preliminary version of a paper that will appear in

Proceedings of the International Conference on Computational Methods in Systems Biology, CMSB06, Lecture Notes in Bioinformatics, 4210:108-126, 2006. 


\begin{abstract}
Membrane systems were introduced as models of computation inspired by the structure and functioning of biological cells. Recently, membrane systems have also been shown to be suitable to model cellular processes. We introduce a new model called Membrane Systems with Peripheral and Integral Proteins. The model has compartments enclosed by membranes, floating objects, objects associated to the internal and external surfaces of the membranes and also objects integral to the membranes. The floating objects can be processed within the compartments and can interact with the objects associated to the membranes. The model can be used to represent cellular processes that involve compartments, surface and integral membrane proteins, transport and processing of chemical substances. As examples we model a circadian clock and the G-protein cycle in yeast saccharomyces cerevisiae and present a quantitative analysis using an implemented simulator.
\end{abstract}

\title{
1 Introduction
}

Membrane systems are models of computation inspired by the structure and the function of biological cells. The model was introduced in 1998 by Gh. Păun and since then many results have been obtained, mostly concerning computational power. A short introductory guide to the field can be found in [12], while an updated bibliography is available via the web-page [18]. Recently (see, e.g., [10]), membrane systems have been successfully applied to systems biology and several models have been proposed for simulating biological processes (e.g., see the monograph dedicated to membrane computing applications $[5])$.

By the original definition, membrane systems are composed of an hierarchical nesting of membranes that enclose regions (the cellular structure), in which free-floating objects (molecules) exist. Each region can have associated rules, called evolution rules, for evolving the free-floating objects and modelling the biochemical reactions present in cell regions. Rules also exist for moving objects across membranes, called symport and antiport rules, modelling cellular transport. Recently, inspired by brane calculus [3], a model of a membrane system, having free-floating objects and objects attached to the membranes, was introduced in [2]. The attached objects model the proteins that are embedded in lipid bilayer cell membranes. In [2], however, objects are associated to an indivisible membrane which has no concept of inner or outer surface, while in [4] objects (peripheral proteins) are attached to either side of a membrane. In reality, many biological processes are driven and controlled by the presence of specific proteins on the appropriate side of and integral to the membrane: there is a constant interaction between floating chemicals and embedded proteins and between peripheral and integral proteins (see, e.g., [1]). Receptor-mediated processes, such as endocytosis (illustrated in Figure 1) and signalling, are crucial to cell function and by definition are critically dependent on the presence of peripheral and integral membrane proteins.

One model of the cell is that of compartments and sub-compartments in constant communication, with molecules being passed from donor compartments to target compartments by interaction with membrane proteins. Once transported to the correct 


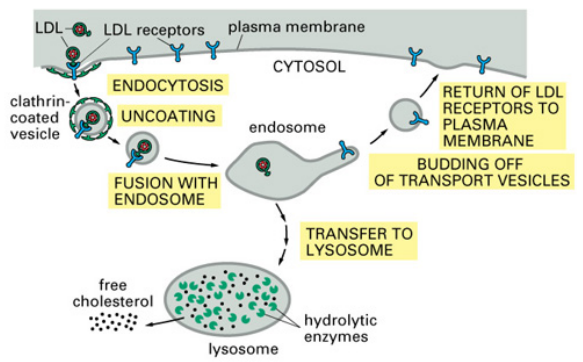

Figure 1: Endocytosis of LDL (Essential Cell Biology, 2/e, (C)2004 Garland Science)

compartment, the substances are then processed by means of local biochemical reactions.

Motivated by these ideas we extend the model presented in [4], introducing a model having peripheral as well as integral proteins.

In each region of the system there are floating objects (the floating chemicals) and, in addition, objects can be associated to each side of a membrane or integral to the membrane (the peripheral and integral membrane proteins). Moreover, the system can perform the following operations: $(i)$ the floating objects can be processed/changed inside the regions of the system (emulating biochemical rules) and (ii) the floating and attached objects can be processed/changed when they interact (modelling the interactions of the floating molecules with membrane proteins).

The proposed model can be used to represent cellular processes that involve floating molecules, surface and integral membrane proteins, transport of molecules across membranes and processing of molecules inside the compartments. As examples, we model a circadian clock and the G-protein cycle in saccharomyces cerevisiae, where the possibility to use, in an explicit way, compartments, membrane proteins and transport rules is very useful. A quantitative analysis of the models is also presented, performed using an extended version of the simulator presented in [4] (downladable at [19]). The simulator employs a stochastic algorithm and uses intuitive syntax based on chemical equations (described in appendix B).

\section{Formal Language Preliminaries}

Membrane systems are based on formal language theory and multiset rewriting. We now briefly recall the basic theoretical notions used in this paper. For more details the reader can consult standard books, such as [8], [15], [6] and handbook [14].

Given the set $A$ we denote by $|A|$ its cardinality and by $\emptyset$ the empty set. We denote by $\mathbb{N}$ and by $\mathbb{R}$ the set of natural and real numbers, respectively.

As usual, an alphabet $V$ is a finite set of symbols. By $V^{*}$ we denote the set of all strings over $V$. By $V^{+}$we denote the set of all strings over $V$ excluding the empty string. The empty string is denoted by $\lambda$. The length of a string $v$ is denoted by $|v|$. The concatenation of two strings $u, v \in V^{*}$ is written $u v$.

The number of occurrences of the symbol $a$ in the string $w$ is denoted by $|w|_{a}$. 
A multiset is a set where each element may have a multiplicity. Formally, a multiset over a set $V$ is a map $M: V \rightarrow \mathbb{N}$, where $M(a)$ denotes the multiplicity of the symbol $a \in V$ in the multiset $M$.

For multisets $M$ and $M^{\prime}$ over $V$, we say that $M$ is included in $M^{\prime}$ if $M(a) \leq M^{\prime}(a)$ for all $a \in V$. Every multiset includes the empty multiset, defined as $M$ where $M(a)=0$ for all $a \in V$.

The sum of multisets $M$ and $M^{\prime}$ over $V$ is written as the multiset $\left(M+M^{\prime}\right)$, defined by $\left(M+M^{\prime}\right)(a)=M(a)+M^{\prime}(a)$ for all $a \in V$. The difference between $M$ and $M^{\prime}$ is written as $\left(M-M^{\prime}\right)$ and defined by $\left(M-M^{\prime}\right)(a)=\max \left\{0, M(a)-M^{\prime}(a)\right\}$ for all $a \in V$. We also say that $\left(M+M^{\prime}\right)$ is obtained by adding $M$ to $M^{\prime}$ (or viceversa) while $\left(M-M^{\prime}\right)$ is obtained by removing $M^{\prime}$ from $M$. For example, given the multisets $M=\{a, b, b, b\}$ and $M^{\prime}=\{b, b\}$, we can say that $M^{\prime}$ is included in $M$, that $\left(M+M^{\prime}\right)=\{a, b, b, b, b, b\}$ and that $\left(M-M^{\prime}\right)=\{a, b\}$.

If the set $V$ is finite, e.g. $V=\left\{a_{1}, \ldots, a_{n}\right\}$, then the multiset $M$ can be explicitly described as $\left\{\left(a_{1}, M\left(a_{1}\right)\right),\left(a_{2}, M\left(a_{2}\right)\right), \ldots,\left(a_{n}, M\left(a_{n}\right)\right)\right\}$. The support of a multiset $M$ is defined as the set $\operatorname{supp}(M)=\{a \in V \mid M(a)>0\}$. A multiset is empty (hence finite) when its support is empty (also finite).

A compact notation can be used for finite multisets: if $M=\left\{\left(a_{1}, M\left(a_{1}\right)\right),\left(a_{2}, M\left(a_{2}\right)\right)\right.$, $\left.\ldots,\left(a_{n}, M\left(a_{n}\right)\right)\right\}$ is a multiset of finite support, then the string $w=a_{1}^{M\left(a_{1}\right)} a_{2}^{M\left(a_{2}\right)} \ldots a_{n}^{M\left(a_{n}\right)}$ (and all its permutations) precisely identify the symbols in $M$ and their multiplicities. Hence, given a string $w \in V^{*}$, we can say that it identifies a finite multiset over $V$, written as $M(w)$, where $M(w)=\left\{a \in V \mid\left(a,|w|_{a}\right)\right\}$. For instance, the string bab represents the multiset $M(w)=\{(a, 1),(b, 2)\}$, that is the multiset $\{a, b, b\}$. The empty multiset is represented by the empty string $\lambda$.

\section{Operations with Peripheral and Integral Proteins}

Let $V$ denote a finite alphabet of objects and $L a b$ a finite set of labels.

As is usual in the membrane systems field, a membrane is represented by a pair of square brackets, []. A membrane structure is an hierarchical nesting of membranes enclosed by a main membrane called the root membrane. To each membrane is associated a label that is written as a superscript of the membrane, e.g. [ ] ${ }^{1}$. If a membrane has the label $i$ we call it membrane $i$.

A membrane structure is essentially that of a tree, where the nodes are the membranes and the arcs represent the containment relation. In this paper we avoid a formal mapping in the interest of the intuitiveness of the description, however, being a tree, a membrane structure can be represented by a string of matching square brackets, e.g., $\left[\left[[]^{2}\right]^{1}[]^{3}\right]^{0}$.

To each membrane there are associated three multisets, $u, v$ and $x$ over $V$, denoted by []$_{u|v| x}$. We say that the membrane is marked by $u, v$ and $x ; x$ is called the external marking, $u$ the internal marking and $v$ the integral marking of the membrane. In general, we refer to them as markings of the membrane.

The internal, external and integral markings of a membrane model the proteins attached to the internal surface, attached to the external surface and integral to the mem- 
brane, respectively.

In a membrane structure, the region between membrane $i$ and any enclosed membranes is called region $i$. To each region is associated a multiset of objects $w$ called the free objects of the region. The free objects are written between the brackets enclosing the regions, e.g., $\left[a a[b b]^{1}\right]^{0}$.

The free objects of a membrane model the floating chemicals within the regions of a cell.

We denote by $\operatorname{int}(i), \operatorname{ext}(i)$ and $\operatorname{itgl}(i)$ the internal, external and integral markings of membrane $i$, respectively. By free $(i)$ we denote the free objects of region $i$. For any membrane $i$, distinct from a root membrane, we denote by out $(i)$ the label of the membrane enclosing membrane $i$.

For example, the string

$$
\left[a b[c c]_{a||}^{2}[a b b]_{b b a|a b| c}^{1}\right]^{0}
$$

represents a membrane structure, where to each membrane are associated markings and to each region are associated free objects. Membrane 1 is internally marked by bba (i.e., $\operatorname{int}(1)=b b a)$, has integral marking $a b$ (i.e., $\operatorname{itgl}(1)=a b$ ) and is externally marked by $c$ (i.e., $\operatorname{ext}(1)=c$ ). To region 1 are associated the free objects $a b b$ (i.e., free $(1)=a b b$ ). To region 0 are associated the free objects $a b$. Finally, out $(1)=$ out $(2)=0$. Membrane 0 is the root membrane. The string can also be depicted diagrammatically, as in Figure 2.

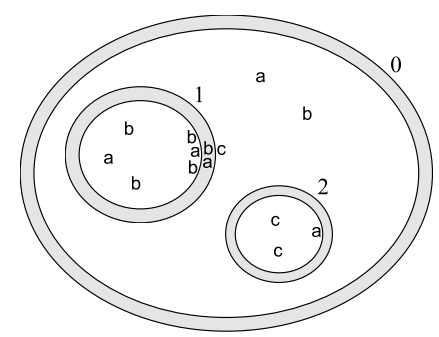

Figure 2: Graphical representation of $\left[a b[c c]_{a||}^{2}[a b b]_{b b a|a b| c}^{1}\right]^{0}$

When a marking is omitted it is intended that the membrane is marked by the empty string $\lambda$, i.e., the empty multiset. For instance, in $[a b]_{u|v|}$ the external marking is missing, while in the case of $[a b]_{|v| x}$ the internal marking is missing.

\subsection{Operations}

We introduce rules that describe bidirectional interactions of floating objects with the membrane markings which we call membrane rules. These rules are motivated by the behaviour of cell membrane proteins (e.g., see [1]) and therefore permit a level of abstraction based on the behaviour of real molecules. We denote the rules as attach ${ }_{\text {in }}$ attach $_{\text {out }}$, de - attach $_{\text {in }}$ and de - attach ${ }_{\text {out }}$, defined: 


$$
\begin{aligned}
& \text { attach }_{\text {in }}:[\alpha]_{u|v|}^{i} \rightarrow[]_{u^{\prime}\left|v^{\prime}\right|}^{i}, \quad \alpha \in V^{+}, u, v, u^{\prime}, v^{\prime} \in V^{*}, i \in L a b \\
& \text { attach }_{\text {out }}:[]_{|v| x}^{i} \alpha \rightarrow[]_{\left|v^{\prime}\right| x^{\prime}}^{i}, \quad \alpha \in V^{+}, v, x, v^{\prime}, x^{\prime} \in V^{*}, i \in \text { Lab } \\
& d e-\text { attach }_{i n}:[]_{u|v|}^{i} \rightarrow[\alpha]_{u^{\prime}\left|v^{\prime}\right|}^{i}, \quad \alpha, u^{\prime}, v^{\prime}, u, v \in V^{*},|u v|>0, i \in L a b \\
& d e-\text { attach }_{\text {out }}:[]_{|v| x}^{i} \rightarrow[]_{\left|v^{\prime}\right| x^{\prime}}^{i} \alpha, \quad \alpha, v^{\prime}, x^{\prime}, v, x \in V^{*},|v x|>0, i \in L a b
\end{aligned}
$$

The semantics of these rules is as follows.

The attach $_{\text {in }}$ rule is applicable to membrane $i$ if $f r e e(i)$ includes $\alpha$, int $(i)$ includes $u$ and $i \operatorname{tgl}(i)$ includes $v$. When the rule is applied to membrane $i, \alpha$ is removed from free $(i), u$ is removed from $\operatorname{int}(i), v$ is removed from $\operatorname{itgl}(i), u^{\prime}$ is added to $\operatorname{int}(i)$ and $v^{\prime}$ is added to $\operatorname{itgl}(i)$. The objects not involved in the application of the rule are left unchanged in their original positions.

The attach $_{\text {out }}$ rule is applicable to membrane $i$ if $f r e e(\operatorname{out}(i))$ includes $\alpha, \operatorname{itgl}(i)$ includes $v, \operatorname{ext}(i)$ includes $x$. When the rule is applied to membrane $i, \alpha$ is removed from free $($ out $(i)), v$ is removed from $\operatorname{itgl}(i), x$ is removed from $\operatorname{ext}(i), v^{\prime}$ is added to $\operatorname{itgl}(i)$ and $x^{\prime}$ is added to $\operatorname{ext}(i)$. The objects not involved in the application of the rule are left unchanged in their original positions.

The de - attach $_{\text {in }}$ rule is applicable to membrane $i$ if $\operatorname{int}(i)$ includes $u$ and $\operatorname{itgl}(i)$ includes $v$. When the rule is applied to membrane $i, u$ is removed from $\operatorname{int}(i), v$ is removed from $\operatorname{itgl}(i), u^{\prime}$ is added to $\operatorname{int}(i), v^{\prime}$ is added to $\operatorname{itgl}(i)$ and $\alpha$ is added to free $(i)$. The objects not involved in the application of the rule are left unchanged in their original positions.

The de - attach $_{\text {out }}$ rule is applicable to membrane $i$ if $i \operatorname{tgl}(i)$ includes $v$ and $\operatorname{ext}(i)$ includes $x$. When the rule is applied to membrane $i, v$ is removed from $\operatorname{itgl}(i), x$ is removed from $\operatorname{ext}(i), v^{\prime}$ is added to $\operatorname{itgl}(i), x^{\prime}$ is added to $\operatorname{ext}(i)$ and $\alpha$ is added to free $($ out $(i))$. The objects not involved in the application of the rule are left unchanged in their original positions.

We denote by $\mathcal{R}_{V, L a b}^{a t t}$ the set of all possible attach and de - attach rules over the alphabet $V$ and set of labels $L a b$. Instances of attach $_{\text {in }}$, attach $_{\text {out }}$, de - attach $_{\text {in }}$ and de - attach $_{\text {out }}$ rules are depicted in Figure 3.

We next introduce evolution rules that rewrite the free objects contained in a region conditional on the markings of the enclosing membrane. These rules can be considered to model the biochemical reactions that take place within the cytoplasm of a cell. We define an evolution rule:

$$
\text { evol : }[\alpha \rightarrow \beta]_{u|v|}^{i}
$$

where $u, v, \beta \in V^{*}, \alpha \in V^{+}$, and $i \in L a b$.

The semantics of the rule is as follows. The rule is applicable to region $i$ if $\operatorname{free}(i)$ includes $\alpha, \operatorname{int}(i)$ includes $u$ and $\operatorname{itgl}(i)$ includes $v$. When the rule is applied to region 

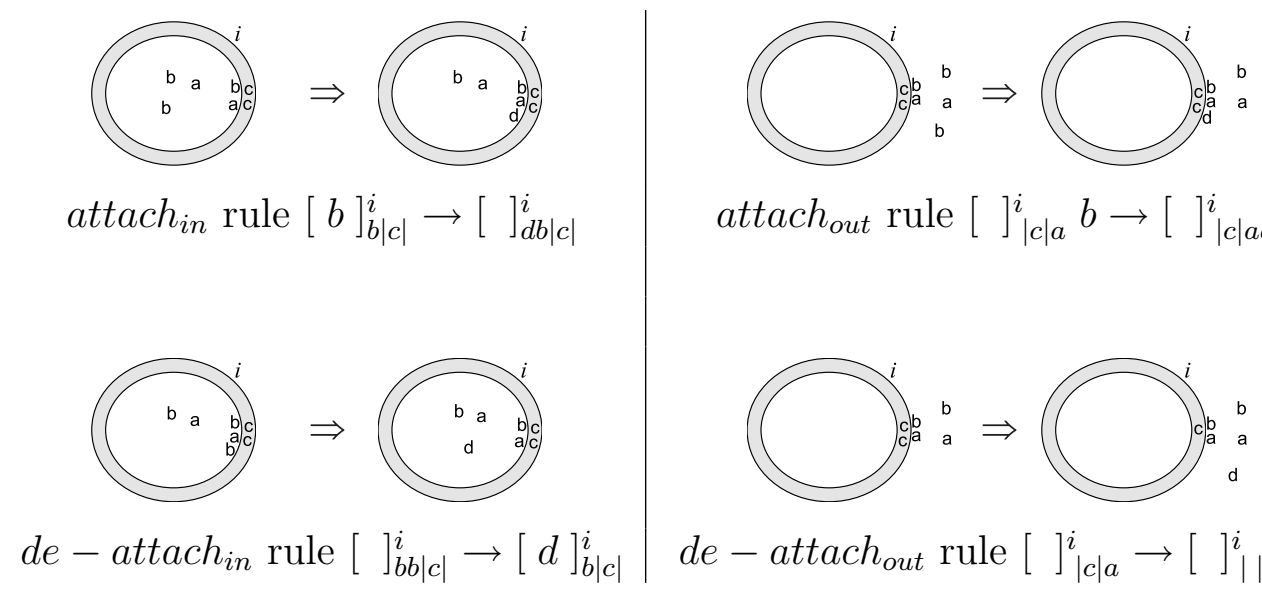

attach $_{\text {out }}$ rule []$_{|c| a}^{i} b \rightarrow[]_{|c| a d}^{i}$

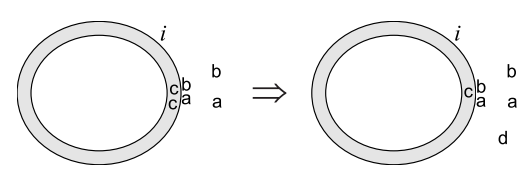

de - attach $_{\text {out }}$ rule []$_{|c| a}^{i} \rightarrow[]_{|| a}^{i} d$

Figure 3: Examples of attach $_{\text {in }}$, ttach $_{\text {out }}$, de-attach ${ }_{\text {in }}$ and de-attach out $_{\text {rules, showing }}$ how free and attached objects may be rewritten. E.g., in the attach $_{\text {in }}$ rule one of the two free instances of $b$ is rewritten to $d$ and added to the membrane's internal marking.

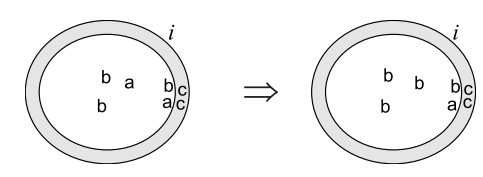

Figure 4: evol rule $[a \rightarrow b]_{b|c|}^{i}$. Free objects can be rewritten inside the region and the rewriting can depend on the integral and internal markings of the enclosing membrane.

$i, \alpha$ is removed from free $(i)$ and $\beta$ is added to free $(i)$. The membrane markings and the objects not involved in the application of the rule are left unchanged in their original positions.

We denote by $\mathcal{R}_{V, L a b}^{e v}$ the set of all evolution rules over the alphabet $V$ and set of labels $L a b$. An instance of an evolution rule is represented in Figure 4.

In general, when a rule has label $i$ we say that a rule is associated to membrane $i$ (in the case of attach and de - attach rules) or is associated to region $i$ (in the case of evol rules). For instance, in Figure 3 the attach $_{\text {in }}$ is associated to membrane $i$.

The objects of $\alpha, u$ and $v$ for attach $_{\text {in }} /$ evol rules, of $\alpha, v$ and $x$ for attach $_{\text {out }}$ rules, of

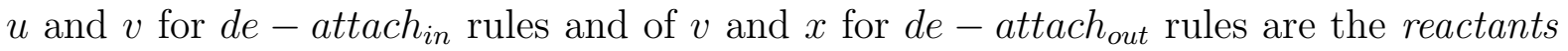
of the corresponding rules. E.g., in the attach rule $[b]_{a|c|} \rightarrow[]_{d|c|}$, the reactants are $a$, $b$ and $c$.

We note that a single application of an evol rule may be simulated by an application of an attach $_{\text {in }}$ rule followed by an application of an de - attach in rule. This may be biologically realistic in some cases, but not in all. Hence the need for evolution rules. 


\section{Membrane Systems with Peripheral and Integral Proteins}

In this section we define membrane systems having membranes marked with peripheral proteins, integral proteins, free objects and using the operations introduced in Section 3.

Definition 4.1 A membrane system with peripheral and integral proteins and $n$ membranes (in short, a $P_{p i}$ system), is a construct

$\mathcal{P}=\left(V_{\mathcal{P}}, \mu_{\mathcal{P}},\left(u_{0}, v_{0}, x_{0}\right)_{\mathcal{P}}, \ldots,\left(u_{n-1}, v_{n-1}, x_{n-1}\right)_{\mathcal{P}}, w_{0, \mathcal{P}}, \ldots, w_{n-1, \mathcal{P}}, R_{\mathcal{P}}, t_{i n, \mathcal{P}}, t_{f i n, \mathcal{P}}\right.$, rate $\left._{\mathcal{P}}\right)$

- $V_{\mathcal{P}}$ is a finite, non-empty alphabet of objects.

- $\mu_{\mathcal{P}}$ is a membrane structure with $n \geq 1$ membranes injectively labelled by labels in $\mathrm{Lab}_{\mathcal{P}}=\{0,1, \cdots, n-1\}$, where 0 is the label of the root membrane.

- $\left(u_{0}, v_{0}, x_{0}\right)_{\mathcal{P}}=(\lambda, \lambda, \lambda),\left(u_{1}, v_{1}, x_{1}\right)_{\mathcal{P}}, \cdots,\left(u_{n-1}, v_{n-1}, x_{n-1}\right)_{\mathcal{P}} \in V^{*} \times V^{*} \times V^{*}$ are called initial markings of the membranes.

- $w_{0, \mathcal{P}}, w_{1, \mathcal{P}}, \cdots, w_{n-1, \mathcal{P}} \in V^{*}$ are called initial free objects of the regions.

- $R_{\mathcal{P}} \subseteq \mathcal{R}_{V, L a b_{\mathcal{P}}-\{0\}}^{a t t} \cup \mathcal{R}_{V, L a b_{\mathcal{P}}}^{e v}$ is a finite set of evolution rules, attach/de-attach rules. ${ }^{1}$

- $t_{i n, \mathcal{P}}, t_{f i n, \mathcal{P}} \in \mathbb{R}$ are called the initial time and the final time, respectively.

- rate $_{\mathcal{P}}: R_{\mathcal{P}} \longmapsto \mathbb{R}$ is the rate mapping. It associates to each rule a rate.

Let $\Pi$ be an arbitrary $P_{p i}$ system. An instantaneous description $I$ of $\Pi$ consists of the membrane structure $\mu_{\Pi}$ with markings associated to the membranes and free objects associated to the regions. We denote by $\mathbb{I}(\Pi)$ the set of all instantaneous descriptions of $\Pi$. We say in short membrane (region) $i$ of $I$ to denote the membrane (region, respectively) $i$ present in $I$.

Let $I$ be an arbitrary instantaneous description from $\mathbb{I}(\Pi)$ and $r$ an arbitrary rule from $R_{\Pi}$. Suppose that $r$ is associated to membrane $i \in L a b_{\Pi}$ if $r \in \mathcal{R}_{V, L a b_{\Pi}-\{0\}}^{a t t}$ (or to region $i \in L a b_{\Pi}$ if $\left.r \in \mathcal{R}_{V, L a b_{\Pi}}^{e v}\right)$.

Then, if $r$ is applicable to membrane $i$ (or to region $i$, accordingly) of $I$, in short we say that $r$ is applicable to $I$. We denote by $r(I) \in \mathbb{I}(\Pi)$ the instantaneous description of $\Pi$ obtained when the rule $r$ is applied to membrane $i$ (or to region $i$, accordingly) of $I$ (in short, we say $r$ is applied to $I$ ).

The initial instantaneous description of $\Pi, I_{i n, \Pi} \in \mathbb{I}(\Pi)$, consists of the membrane structure $\mu_{\Pi}$ with membrane $i$ marked by $\left(u_{i}, v_{i}, x_{i}\right)_{\Pi}$ for all $i \in L a b_{\Pi}-\{0\}$ and free objects $w_{i, \Pi}$ associated to region $i$ for all $i \in L a b_{\Pi}$.

\footnotetext{
${ }^{1}$ The root membrane may contain objects and evolution rules but not attach or de - attach rules, since it has no enclosing region. It may therefore be viewed as an extended version of a membrane systems environment (as defined in [12]), with objects and evol rules. Alternatively, it can be seen as a membrane systems skin membrane, where the environment contains nothing and is not accessible.
} 
A configuration of $\Pi$ is a pair $(I, t)$ where $I \in \mathbb{I}(\Pi)$ and $t \in \mathbb{R} ; t$ is called the time of the configuration. We denote by $\mathcal{C}(\Pi)$ the set of all configurations of $\Pi$. The initial configuration of $\Pi$ is $C_{i n, \Pi}=\left(I_{i n, \Pi}, t_{i n, \Pi}\right)$.

Suppose that $R_{\Pi}=\left\{\right.$ rule $^{1}$, rule $^{2}, \ldots$, rule $\left.^{m}\right\}$ and let $S$ be an arbitrary sequence of configurations $\left\langle C_{0}, C_{1}, \cdots, C_{j}, C_{j+1}, \cdots, C_{h}\right\rangle$, where $C_{j}=\left(I_{j}, t_{j}\right) \in \mathcal{C}(\Pi)$ for $0 \leq j \leq h$. Let $a_{j}=\sum_{i=1}^{m} p_{j}^{i}, 0 \leq j \leq h$, where $p_{j}^{i}$ is the product of rate $\left(\right.$ rule $\left.{ }^{i}\right)$ and the mass action combinatorial factor for rule and $I_{j}$ (see Appendix A).

The sequence $S$ is an evolution of $\Pi$ if

- for $j=0, C_{j}=C_{i n, \Pi}$

- for $0 \leq j \leq h-1, a_{j}>0, C_{j+1}=\left(r_{j}\left(I_{j}\right), t_{j}+d t_{j}\right)$ with $r_{j}, d t_{j}$ as in [7]:

$$
\begin{aligned}
& -r_{j}=\operatorname{rule}^{k}, k \in\{1, \cdots, m\} \text { and } k \text { satisfies } \sum_{i=1}^{k-1} p_{j}^{i}<r a n_{j}^{\prime} \cdot a_{j} \leq \sum_{i=1}^{k} p_{j}^{i} \\
& -d t_{j}=\left(-1 / a_{j}\right) \ln \left(\operatorname{ran}_{j}^{\prime \prime}\right)
\end{aligned}
$$

where $\operatorname{ran}_{j}^{\prime}, \operatorname{ran}_{j}^{\prime \prime}$ are two random variables over the sample space $(0,1]$, uniformly distributed.

- for $j=h, a_{j}=0$ or $t_{j} \geq t_{f i n, \Pi}$.

In other words, an evolution of $\Pi$ is a sequence of configurations, starting from the initial configuration of $\Pi$, where, given the current configuration $C_{j}=\left(I_{j}, t_{j}\right)$, the next one, $C_{j+1}=$ $\left(I_{j+1}, t_{j+1}\right)$, is obtained by applying the rule $r_{j}$ to the current instantaneous description $I_{j}$ and adding $d t_{j}$ to the current time $t_{j}$. The rule $r_{j}$ is applied as described in Section 3. Rule $r_{j}$ and $d t_{j}$ are obtained using the Gillespie algorithm [7] over the current instantaneous description $I_{j}$. The evolution halts when all rules have zero probability of being applied $\left(a_{j}=0\right)$ or when the current time is greater or equal to the specified final time.

\section{Modelling and Simulation of Cellular Processes}

Having established a theoretical basis, we now wish to demonstrate the quantitative behaviour of the presented model. To this end we have extended the simulator presented in [4] to produce evolutions of an arbitrary $P_{p i}$ system. In Sections 5.2 and 5.3 we demonstrate the model and the simulator using two examples from the literature.

\subsection{The Stochastic Algorithm}

We use a discrete stochastic algorithm based on Gillespie's which can more accurately represent the dynamical behaviour of small quantities of reactants, in comparison, say, to a deterministic approach based on ordinary differential equations [11]. Moreover, Gillespie has shown that the algorithm is fully equivalent to the chemical master equation. 
The Gillespie algorithm is specifically designed to model the interaction of chemical species and imposes a restriction of a maximum of three reacting molecules. This is on the basis that the likelihood of more than three molecules colliding is vanishingly small. Hence the simulator is similarly restricted. Note that in the evolution of a $P_{p i}$ system, the stochastic algorithm does not distinguish between floating objects and objects attached or integral to the membrane. That is, the algorithm is applied to the objects irrespective of where they are in the compartment on the assumption that the interaction between floating and attached molecules can be considered the same as between floating molecules. Our application of the Gillespie algorithm to membranes is further described in Appendix A.

\subsection{Modelling a Noise-Resistant Circadian Clock}

Many organisms use circadian clocks to synchronise their metabolisms to a daily rhythm, however the precise mechanisms of implementation vary from species to species. One common requirement is the need to maintain a measure of stability of timing in the face of perturbations of the system: the clock must continue to tick and keep good time. A general model which captures the essence of such stability, based on common elements of several real biological clocks, is presented in [16]. We choose this as an interesting, non-trivial example to model and simulate with a $P_{p i}$ system using evolution rules alone. Moreover, we choose this example because it has been modelled in other formalisms, such as in stochastic $\Pi$ calculus (see, e.g., [17], [13]).

The model is described diagrammatically in Figure 5. The system consists of two different genes ( $\mathrm{gA}$ and $\mathrm{gR}$ ) which produce two different proteins (pA and $\mathrm{pR}$, respectively) via two different mRNA species ( $\mathrm{mA}$ and $\mathrm{mR}$, respectively). Protein $\mathrm{pA}$ up-regulates the transcription of its own gene and also the transcription of the gene that produces $\mathrm{pR}$. The proteins are removed from the system by simple degradation to nothing (dashed lines) and by the formation of a complex AR. In this latter way the production of $\mathrm{pR}$ reduces the concentration of $\mathrm{pA}$ and has the consequence of down-regulating $\mathrm{pR}$ 's own production. Thus, in turn, $\mathrm{pA}$ is able to increase, increasing the production of $\mathrm{pR}$ and causing the cycle to repeat. Key elements of the stable dynamics are the rapid production of pA, by virtue of positive feedback, and the relative rate of growth of the complexation reaction.

A description of the $P_{p i}$ system used to model the circadian clock is given in Figure 6, together with the corresponding simulator script for comparison. The alphabet, $V_{\text {clock}}$, is specified to contain all the reacting species. This corresponds to the object statement of the simulator script. The sixteen chemical reactions of Figure 5 are simply transcribed into corresponding rules mapped to reaction rates. In the simulator script they are grouped under one identifier, clock. The membrane structure, $\mu_{\text {clock }}$, comprises just the root membrane. The root region initially contains one copy each of the two genes as free objects. These facts are reflected in the system statement of the simulator script, which also associates to the contents the set of rules clock.

The results of running the script are shown in Figure 5: the two proteins exhibit anti-phase periodicity of approximately 24 hours, as expected. 

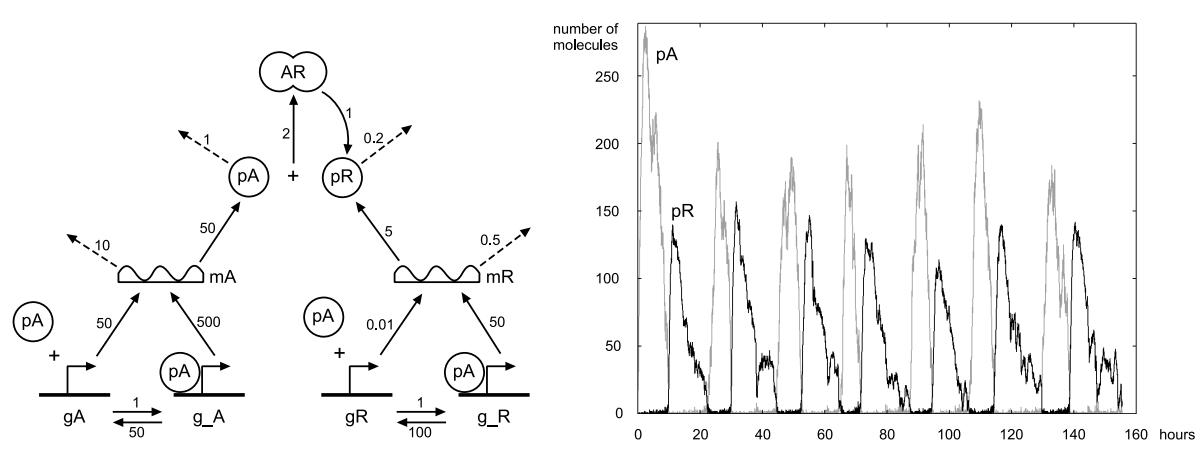

Figure 5: Reaction scheme and simulation results of noise-resistant circadian clock of [16]

The simulator has the capability to add or subtract reactants from the simulation in runtime. We use this facility to discover the effect of switching off $\mathrm{gR}$ in the circadian clock by making the following addition to the system statement:

-1 gR $050000,-1$ g_R $@ 50000$

These instructions request a subtraction from the system at time step 50000 of one gR and one g_R. Note that to switch off the gene it is necessary to remove both versions (i.e., with and without pA bound), since it is not possible to know in what state it will exist at a particular time step. Negative quantities are not allowed in the simulator, so only the existent specie will be deleted. In general, the number subtracted is the minimum of the existent quantity and the requested amount. The same syntax, without the negative sign, is used to add reactants.

The effect of switching off gR, shown in Figure 7, is to reduce the amount of $\mathrm{pR}$ to near zero and to thus allow pA to reach a maximum governed by its relative rates of production and decay. Note that a small amount of $\mathrm{pR}$ continues to exist long after its gene has been switched off. This is the result of a so-called hidden pathway from the AR complex, which decays at a much slower rate than $\mathrm{pR}$ (second graph of Figure 7). Although this model is a generalisation of biological circadian clocks and may not represent the behaviour of a specific example, the existence of an unexpected pathway exemplifies an important problem encountered when attempting to predict the behaviour of biological systems.

\subsection{Modelling Saccharomyces Cerevisiae Mating Response}

To demonstrate the ability of $P_{p i}$ systems to represent compartments and membranes we model and simulate the G-protein mating response in yeast saccharomyces cerevisiae, based on experimental rates provided by [9]. The G-protein transduction pathway involves membrane proteins and the transport of substances between regions and is a mechanism by which organisms detect and respond to environmental signals. It is extensively studied and many pharmaceutical agents are aimed at components of the G-protein cycle in humans. The diagram in Figure 8 shows the relationships between the various reactants and regions modelled and simulated.

A description of the biological process is that the yeast cell receives a signal ligand 

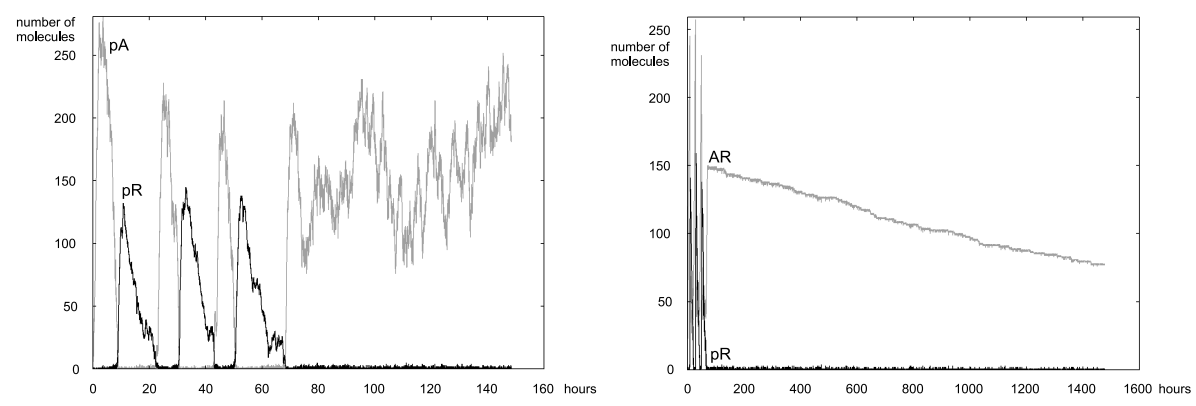

Figure 7: Simulated effect of switching off gA in circadian clock of [16]
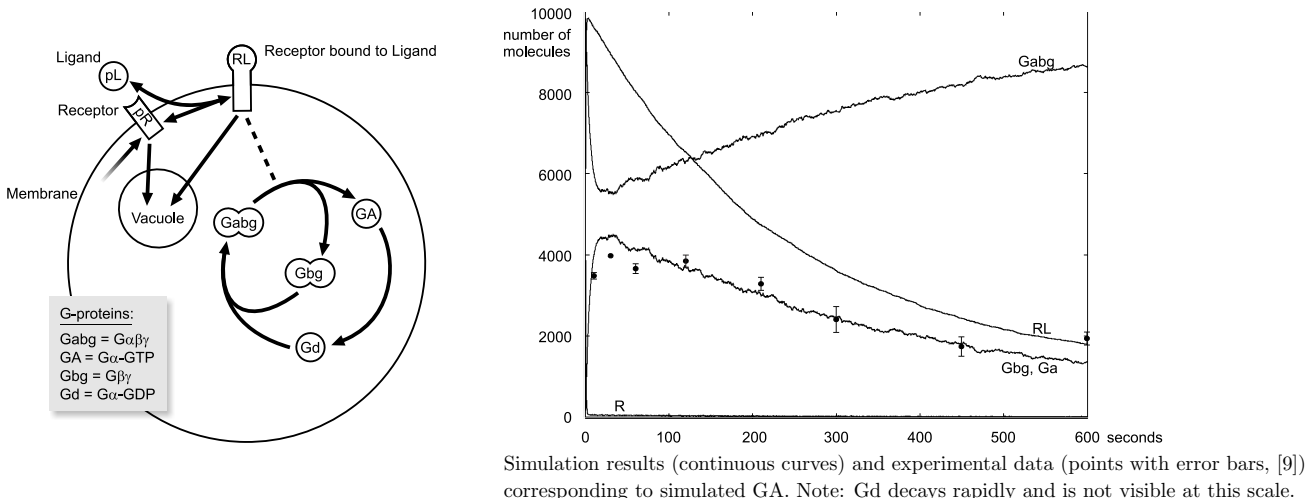

Figure 8: Model and simulation results of saccharomyces cerevisiae mating response.

\section{Prospects}

We have introduced a model of membrane systems (called a $P_{p i}$ system) with objects integral to the membrane and objects attached to either side of the membrane. We have also introduced operations that can rewrite floating objects conditional on the existence of integral and attached objects and operations that facilitate the interaction of floating objects with integral and attached objects. With these we are able to model in detail many real biochemical processes occurring in the cytoplasm and in the cell membrane.

Evolutions of a $P_{p i}$ system are obtained using an algorithm based on Gillespie [7] and in the second part of the paper we have presented a simulator which can produce evolutions of an arbitrary $P_{p i}$ system, using syntax based on chemical equations. To demonstrate the utility of $P_{p i}$ systems and of the simulator we have modelled and simulated a circadian clock and the G-protein cycle mating response of saccharomyces cerevisiae. The latter makes extensive use of membrane operations.

Several different research directions are now proposed. The primary direction is the application of $P_{p i}$ systems and of the simulator to real biological systems, with the aim of prediction by in-silico experimentation. Such application is likely to lead to the need for new bio-inspired features and these constitute another direction of research. The features will be implemented in the model and simulator as necessary, however it is already envisaged that operations of fission and fusion will be required to permit the modification of a membrane structure in runtime. 
[5] G. Ciobanu, Gh. Păun, M.J. Pérez-Jiménez, eds., Applications of Membrane Computing. SpringerVerlag, Berlin, 2006.

[6] J. Dassow, Gh. Păun, Regulated Rewriting in Formal Language Theory. Springer-Verlag, Berlin, 1989.

[7] D. T. Gillespie, A General Method for Numerically Simulating the Stochastic Time Evolution of Coupled Chemical Reactions. Journal of Computational Physics, 22, 1976.

[8] J.E. Hopcroft, J.D. Ullman, Introduction to Automata Theory, Languages, and Computation. Addison-Wesley, 1979.

[9] T.-M. Yi, H. Kitano, M. I. Simon, A quantitative characterization of the yeast heterotrimeric G protein cycle. Proceedings of the National Academy of Science, 100, 19, 2003.

[10] M. J. Pérez-Jiménez, F. J. Romero-Campero, Modelling EGFR signalling network using continuous membrane systems. Proceedings of the Third Workshop on Computational Method in Systems Biology, Edinburgh, 2005.

[11] H. McAdams, A. Arkin, Stochastic mechanisms in gene expression. Proceedings of the National Academy of Science, 94, 1997.

[12] Gh. Păun, G. Rozenberg, A Guide to Membrane Computing. Theoretical Computer Science, 287-1, 2002.

[13] A. Regev, W. Silverman, N. Barkai, E. Shapiro, Computer Simulation of Biomolecular Processes using Stochastic Process Algebra. Poster at 8th International Conference on Intelligent Systems for Molecular Biology, ISMB, 2000.

[14] G. Rozenberg, A. Salomaa, eds., Handbook of Formal Languages. Springer-Verlag, Berlin, 1997.

[15] A. Salomaa, Formal Languages. Academic Press, New York, 1973.

[16] M. G. Vilar, H. Y. Kueh, N. Barkai, S. Leibler, Mechanisms of noise-resistance in genetic oscillators. Proceedings of the National Academy of Science, 99, 9, 2002.

[17] http://www.wisdom.weizmann.ac.il/〜 biospi/

[18] http://ppage.psystems.eu

[19] http://www.cosbi.eu/rpty_soft_cytosim.php

Revised 30/9/09 


\section{Appendices}

\section{A The Gillespie Algorithm Applied To Membranes}

The Gillespie algorithm is an exact stochastic simulation of a 'spatially homogeneous mixture of molecular species which inter-react through a specified set of coupled chemical reaction channels' [7]. It is unclear whether a biological cell contains a spatially homogeneous mixture of molecular species and less clear still whether integral and peripheral proteins can be described in this way, however for the purposes of the $P_{p i}$ system model we choose to regard them as such. Hence we treat the objects attached to the membrane as homogeneously mixed with the floating objects, however objects of the same type (i.e. having the same name) but existing in different regions are considered to be of different types in the stochastic algorithm.

The mass action combinatorial factors of the Gillespie algorithm, defined by equations $(14 \mathrm{a} . \mathrm{g})$ in [7], are calculated over the set of chemical reactions given in equations (2a...g) of [7], using standard stoichiometric syntax of the general form

$$
S_{1}+S_{2}+S_{3} \rightarrow P_{1}+P_{2}+\cdots+P_{n}
$$

$S_{1}, S_{2}$ and $S_{3}$ are the reactants and $P_{1}, \ldots, P_{n}$ are the products of the reaction. Since the order of the reactants and products is unimportant they may be represented as multisets $S_{1} S_{2} S_{3}$ and $P_{1} P_{2} \cdots P_{n}$, respectively, over the set of objects $V$. Hence a chemical reaction may be expressed using the notation

$$
S_{1} S_{2} S_{3} \rightarrow P_{1} P_{2} \cdots P_{n}
$$

In the definition of the evolution of a $P_{p i}$ system, the mass action combinatorial factor is calculated using equations (14a...g)[7] after transforming the membrane and evolution rules into chemical reactions and the objects of the current instantaneous description, using the following procedure.

Let $V_{i}=\left\{a_{i} \mid a \in V\right\}, V_{i, \text { int }}=\left\{a_{i, \text { int }} \mid a \in V\right\}, V_{i, i t g l}=\left\{a_{i, i t g l} \mid a \in V\right\}$ and $V_{i, \text { out }}=$ $\left\{a_{i, \text { out }} \mid a \in V\right\}$. We then define morphisms free ${ }^{i}: V \rightarrow V_{i}$, int ${ }^{i}: V \rightarrow V_{i, \text { int }}$, itgl ${ }^{i}: V \rightarrow$ $V_{i, i t g l}$ and out ${ }^{i}: V \rightarrow V_{i, o u t}$ such that free $^{i}(a)=a_{i}$, int ${ }^{i}(a)=a_{i, \text { int }}$, itgl $l^{i}(a)=a_{i, i t g l}$ and out $^{i}(a)=a_{i, \text { out }}$ for $a \in V$. Hence we map an evolution rule of the type

$$
[\alpha \rightarrow \beta]_{u|v|}^{i}
$$

with $u, v, \alpha, \beta \in V^{*}$ and $i \in L a b$, to the chemical reaction

$$
\operatorname{free}^{i}(\alpha) \cdot \operatorname{int}^{i}(u) \cdot i \operatorname{igl} l^{i}(v) \rightarrow \operatorname{free}^{i}(\beta) \cdot \operatorname{int}^{i}(u) \cdot i \operatorname{tgl} l^{i}(v)
$$

We map membrane rules, generally described by

$$
[\alpha]_{u|v| x}^{i} \beta \rightarrow\left[\alpha^{\prime}\right]_{u^{\prime}\left|v^{\prime}\right| x^{\prime}}^{i} \beta^{\prime}
$$

with $u, v, x, \alpha, \beta, u^{\prime}, v^{\prime}, x^{\prime}, \alpha^{\prime}, \beta^{\prime} \in V^{*}$ and $i \in L a b$, to the chemical equation 


$$
\begin{aligned}
& \operatorname{free}^{i}(\alpha) \cdot \operatorname{int}^{i}(u) \cdot \operatorname{itgl}^{i}(v) \cdot \text { out }^{i}(x) \cdot \text { free }^{j}(\beta) \rightarrow \\
& \qquad f r e e^{i}\left(\alpha^{\prime}\right) \cdot \text { int }^{i}\left(u^{\prime}\right) \cdot \operatorname{itgl}^{i}\left(v^{\prime}\right) \cdot \text { out }^{i}\left(x^{\prime}\right) \cdot \text { free }^{j}\left(\beta^{\prime}\right)
\end{aligned}
$$

where $j \in L a b$ is the marking of the membrane surrounding the region enclosing membrane $i$.

The objects of the current instantaneous description are similarly transformed, using the morphisms defined above, in order to correspond with the transformed membrane and evolution rules.

\section{B The Simulator Syntax}

The simulator syntax aims to be an intuitive interpretation of the $P_{p i}$ system model. A simulator script conforms to the following grammar:

$$
\begin{aligned}
\text { SimulatorScript }= & \{\text { Object Declaration, NewLine }\}^{+} \\
& \{\text {Rule Definition, NewLine }\}^{+} \\
& \{\text {Compartment Definition, NewLine }\} \\
& \text { System Statement, NewLine } \\
& \text { Evolve Statement, NewLine } \\
& \text { Plot Statement, }[\text { NewLine }]
\end{aligned}
$$

where NewLine is an appropriate sequence of characters to generate a new line.

An example of a simple simulator script is shown below, together with its $P_{p i}$ system counterpart.

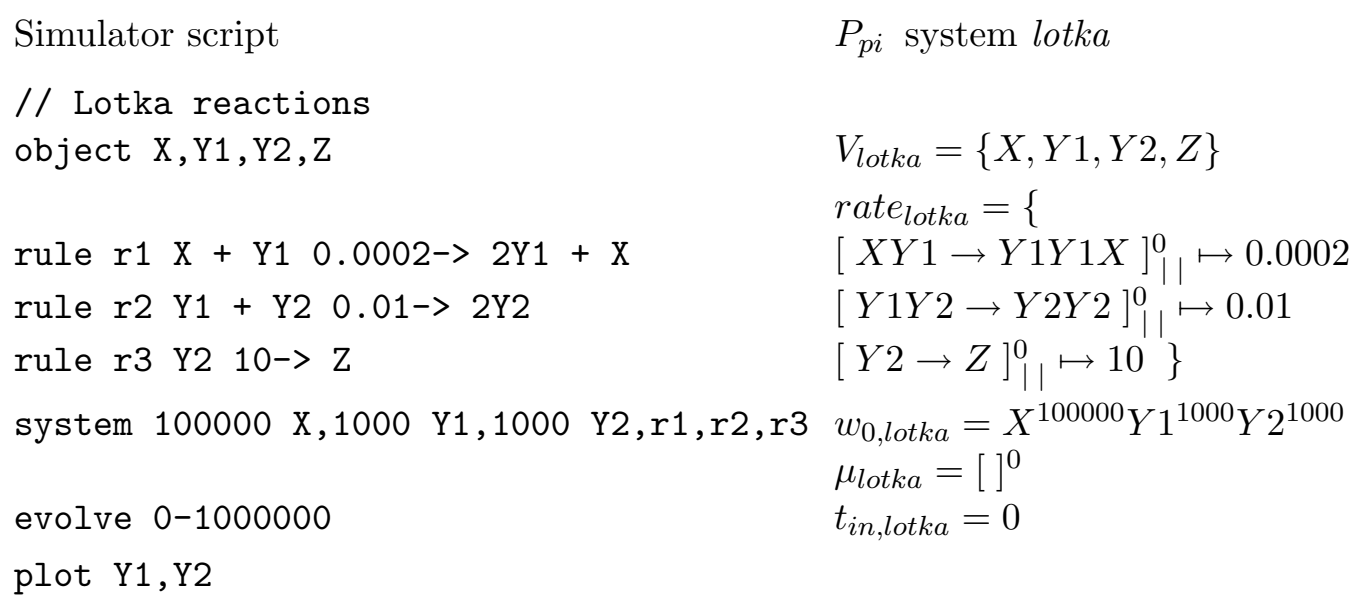

The syntax of the sections of a simulator script are described below.

\section{B.1 Comments}

Comments begin with a double forward slash $(/ /)$ and include all subsequent text on a single line. They may appear anywhere in the script. 


\section{B.2 Object Declaration}

The reacting objects are defined in one or more statements beginning with the keyword object followed by a comma separated list of unique reactant names. E.g.:

object $\mathrm{X}, \mathrm{Y} 1, \mathrm{Y} 2, \mathrm{Z}$

The names are case-sensitive and must start with a letter but may include digits and the underscore character (_). This corresponds to defining the alphabet $V$ of the $P_{p i}$ system.

\section{B.3 Rule Definition}

The reaction rules are defined using rule definitions comprising the keyword rule followed by a unique name and the rewriting rule itself. E.g.:

rule $\mathrm{r} 1 \mathrm{X}+\mathrm{Y} 10.0002->2 \mathrm{Y} 1+\mathrm{X}$

These correspond to the attach / de-attach and evolution rules of the $P_{p i}$ system model. Note, however, that simulator rules are user-defined types which may be instantiated in more than one region. The value preceding the implication symbol $(->)$ is the average reaction rate and corresponds to an element of the range of the mapping rate given in Definition 1. In the simulator it is also possible to define a reaction rate as the product of a constant and the rate of a previously defined rule, using the name of the previous rule in the following way:

rule r2 Y1 + Y2 $50 \mathrm{r} 1->2 \mathrm{Y} 2$

This has the meaning that rule $r 2$ has a rate 50 times that of $r 1$. In addition, in the simulator it is possible to define a group of rules using a single identifier and braces. E.g.,

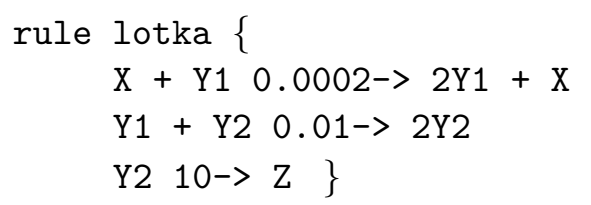

To include membrane operations the simulator rule syntax is extended with the II symbol. Objects listed on the left hand side of the II represent the internal markings, objects listed on the right hand side represent the external markings and objects listed between the vertical bars are the integral markings of the membrane. E.g.:

rule $r 4 \mathrm{X}+|\mathrm{Y} 2| 0.1->|\mathrm{X}, \mathrm{Y} 2|$

means that if one $\mathrm{X}$ exists within the compartment and one $\mathrm{Y} 2$ exists integral to the membrane, then the $\mathrm{X}$ will be added to the integral marking of the membrane. The $P_{p i}$ system equivalent is the following attach $_{\text {in }}$ rule:

$[X]_{|Y 2|} \rightarrow[]_{|X Y 2|}$

To represent an attach $_{\text {out }}$ rule in the simulator the following syntax is used:

rule $\mathrm{r} 4|\mathrm{Y} 2|+\mathrm{X} 0.1->|\mathrm{X}, \mathrm{Y} 2|$

Here the $\mathrm{X}$ appears to the right of the $\mathrm{I} \mid \mathrm{symbol}$ following $\mathrm{a}+$, meaning that it must exist in the region surrounding the membrane for the rule to be applied. Hence the + used in simulator membrane rules is non-commutative. 


\section{B.4 Compartment Definition}

Compartments may be defined using the keyword compartment followed by a unique name and a list of contents and rules, all enclosed by square brackets. For example,

compartment $\mathrm{c} 1$ [100 X, $100 \mathrm{Y} 1, \mathrm{r} 1, \mathrm{r} 2]$

instantiates a compartment having the label c1 containing $100 \mathrm{X}, 100 \mathrm{Y} 1$ and rules $\mathrm{r} 1$ and r2. In a $P_{p i}$ system such a compartment would have a $P_{p i}$ system (partial) initial instantaneous description

$\left[X^{100} Y 1^{100}\right]^{1}$

Note that a $P_{p i}$ system requires a numerical membrane label and that any rules associated to the region or membrane must be defined separately.

Compartments may contain other pre-defined compartments, so the following simulator statement

compartment c2 [100 Y2, c1]

corresponds to the $P_{p i}$ system (partial) initial instantaneous description

$\left[Y 2^{100}\left[X^{100} Y 1^{100}\right]^{1}\right]^{2}$

Membrane markings in the simulator are added to compartment definitions using the symbol $\mathrm{I}$, to the right of and separated from the floating contents by a colon. E.g.,

compartment c3 [100 X, c2 : 10 Y2||10 Y1]

has the meaning that the compartment c3 contains compartment c2, $100 \mathrm{x}$, and the membrane surrounding $\mathrm{c} 3$ has 10 Y2 attached to its inner surface and 10 Y1 attached to its outer surface. This corresponds to the $P_{p i}$ system (partial) initial instantaneous description

$$
\left[X^{100}\left[Y 2^{100}\left[X^{100} Y 1^{100}\right]^{1}\right]^{2}\right]_{Y 2^{10}|| Y 1^{10}}^{3}
$$

\section{B.5 System Statement}

The system is instantiated using the keyword system followed by a comma-separated list of constituents. E.g.:

system $100000 \mathrm{X}, 1000 \mathrm{Y} 1,1000 \mathrm{Y} 2, \mathrm{r} 1, \mathrm{r} 2, \mathrm{r} 3$

This statement corresponds to the definition of $u_{0} \ldots u_{n}, v_{0} \ldots v_{n}, w_{0} \ldots w_{n}, x_{0} \ldots x_{n}$ and $\mu$ of the $P_{p i}$ system.

The system statement may be extended to multiple lines by enclosing the list of constituents between braces. E.g.:

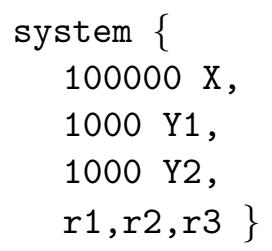

It is also possible to add or subtract reactants from the simulation in runtime using the following syntax in the system statement: 
$-10 \mathrm{X} @ 50000,10 \mathrm{Y} 1 @ 50000$

These instructions request a subtraction of ten $\mathrm{X}$ from the system and an addition of ten Y1 to the system at time step 50000. Negative quantities are not allowed in the simulator, so if a subtraction requests a greater amount than exists, only the existing amount will be deleted.

\section{B.6 Evolve Statement}

The simulator requires a directive to specify the total number of evolution steps to perform and also the number of the evolution step at which to start recording data. This is achieved using the keyword evolve followed by the minimum and maximum evolution steps to record. E.g.,

evolve $0-1000000$

Note that the minimum evolution step does not correspond to $t_{i n}$ of the $P_{p i}$ system, since the simulation always starts from the $0^{\text {th }}$ step. By convention, the simulator sets the initial time of the simulation to 0 , hence $t_{i n}=0$ for all simulations. Note that although $t_{f i n}$ of a $P_{p i}$ system evolution corresponds to the maximum evolution step, the units are different and there is no explicit conversion.

\section{B.7 Plot Statement}

To specify which objects are to be observed during the evolution the plot keyword is used followed by a list of reactants. To plot the contents of a specific compartment the plot statement uses syntax similar to that used in the compartment definition. E.g.,

plot $\mathrm{X}, \mathrm{c} 3[\mathrm{X}, \mathrm{Y} 1: \mathrm{Y} 1|\mathrm{Y} 2|]$

plots the number of free-floating $\mathrm{X}$ in the environment and the specified contents of compartment c3 and its membrane. 